\title{
Architecture for Cognitive Networking within NASA's Future Space Communications Infrastructure
}

\section{Presented By: Gilbert Clark / MTI Systems}

\author{
Authors \\ Gilbert Clark and Wesley M. Eddy/MTI Systems \\ Sandra K. Johnson/NASA Glenn Research Center \\ James Barnes/MTI Systems \\ David Brooks/VPL
}




\section{Presenter Biography}

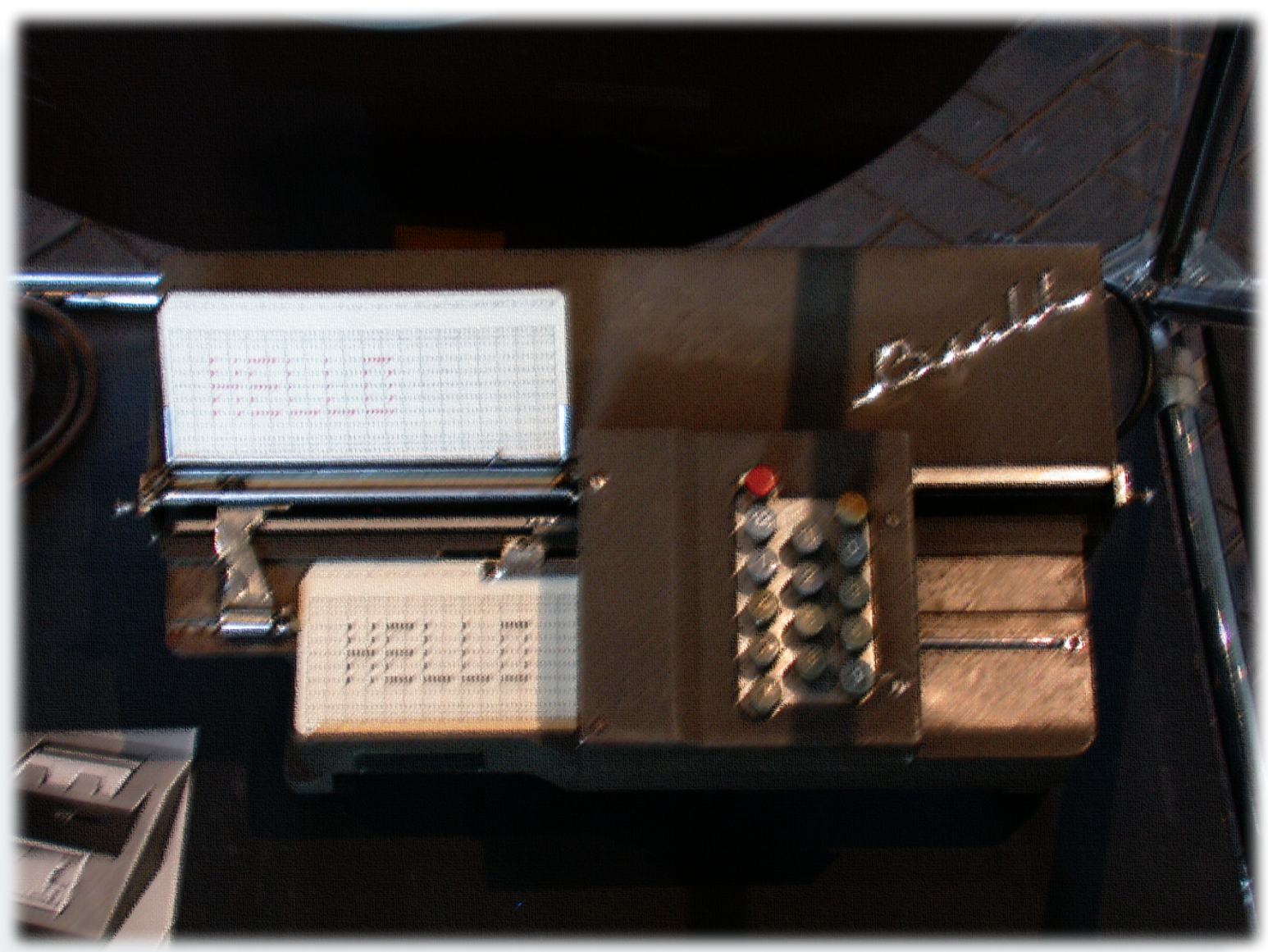




\section{Agenda}

- Discuss cognitive and possible roles in future SCaN

- Discuss architecture concepts

- What might cognitive look like?

- Definitely more than just the radios!

- Describe Cognitive Agent prototype software

- Cognitive networking framework on the SCaN Testbed

- What does future work look like? 


\section{SCaN Future Architecture}

- Moving away from discrete networks

- Space Network, Near Earth Network, Deep Space Network, ...

- Moving toward unified architecture

- Seamless service provision, service interfaces, and scheduling for all network elements

- Cross-layer services: raw signals, bitstreams, link-layer frames, packets, UDP, delay-tolerant networking, ...

- Future solar system internet (SSI) as described by CCSDS

- International, government, and commercial users

- Should all use networks responsibly ...

- Goals for cognitive

- Reduce user burden

- Mitigate operational risks due to growing complexity

- Open-loop communication and navigation services

- Reduce need for direct operator intervention 


\title{
Goals: NASA Intelligent Routing (NITRO) Effort
}

\author{
Reduce operator burden
}

Enhance performance on operational efficiency metrics ...

Improve operator efficiency

Support scale-up in complexity, diversity, and volume / capacity ...

... without a corresponding scale-up in human resource allocations

3 Facilitate autonomous operations

Enable operations where no human can support communications infrastructure (e.g. Mars) 


\section{Iterative Development}

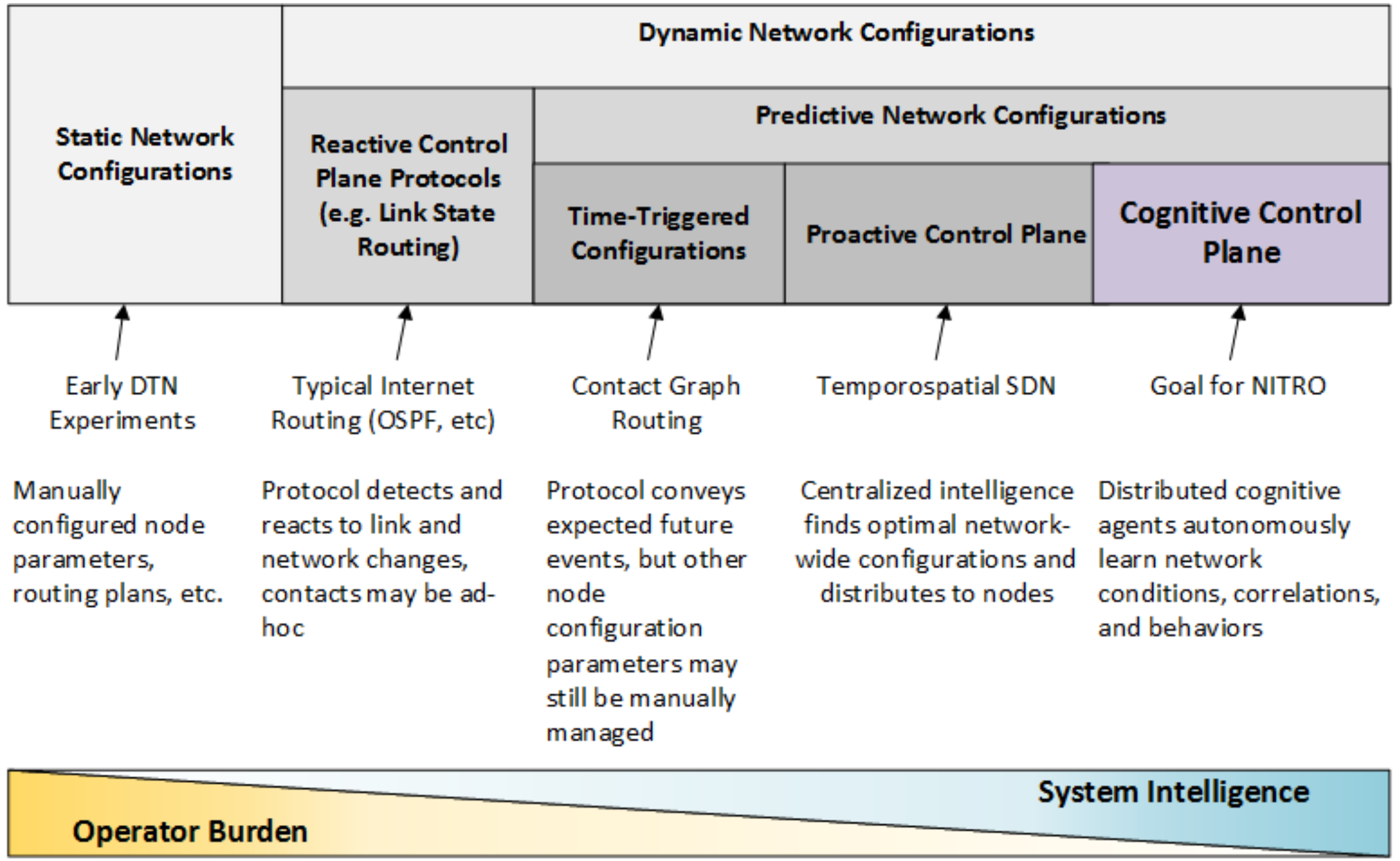




\section{Toward Cognitive System Engineering}

- Not really One True Cognitive to rule them all

- Instead, things are situationally appropriate

- Huge number of different AI and ML techniques

- Neural networks, genetic programming, SVM, and more!

- Different techniques make sense in different situations

- Need to blend autonomy and automation ...

- ... in ways that make sense for the mission

- Many different techniques to achieve cognitive behaviors ...

- Cognitive offload - perform computation elsewhere

- Autonomic computing / networking - "self-management"

- Information-centric networking - emphasize "what", not "where"

- ... optimized across many different "domains"

- "big brain vs. little brain" 


\section{Cognitive Scope}

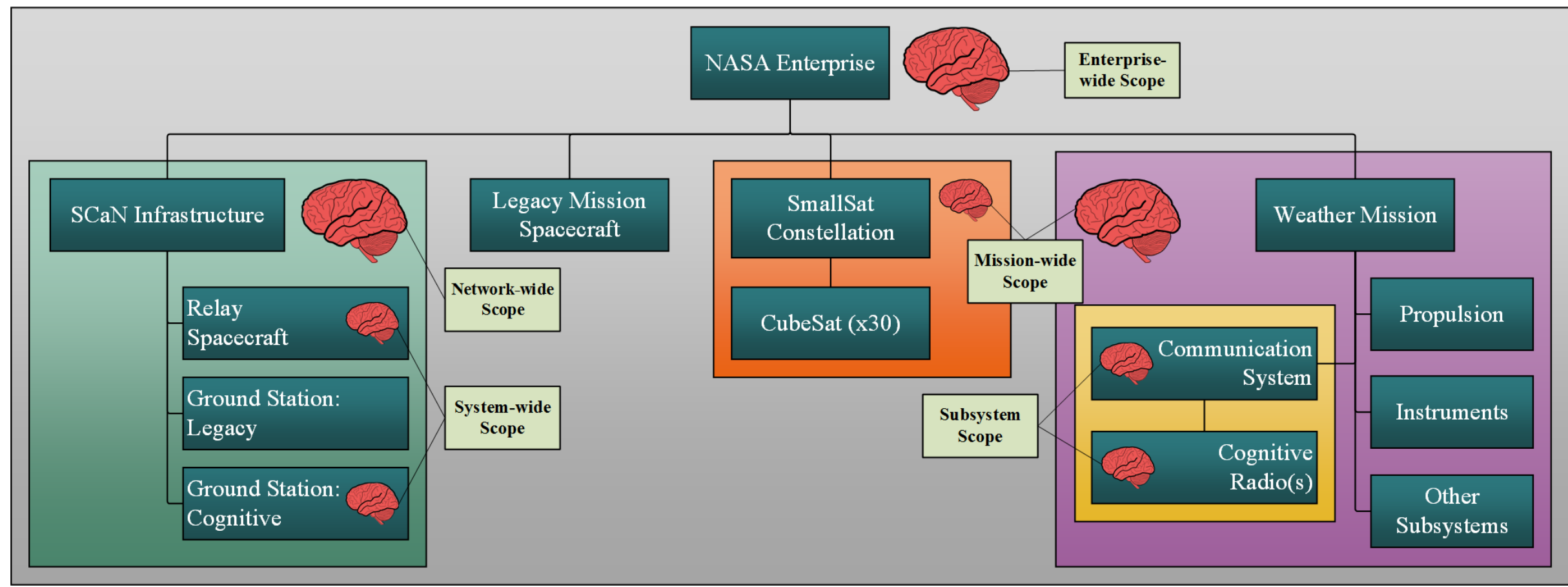




\section{Prototype Cognitive Agent}

- Prototyping intelligent routing software and protocol

- Built to support present and future flight / ground systems

- Current on-orbit testing via SCaN Testbed ...

- Empirically determines link characteristics ...

- ... and makes routing decisions based on goals

- "minimize latency", "maximize reliability", etc.

- Also collects data that can be used for future work ...

- In order to learn, we need data from which to learn ...

- ... and offers a way to swap cognitive engines

- Less of a focus on immediate intelligence in this agent

- More of a focus on a good API and an extensible framework

- Make future experiments easier ... 


\section{Cogent - Construction}

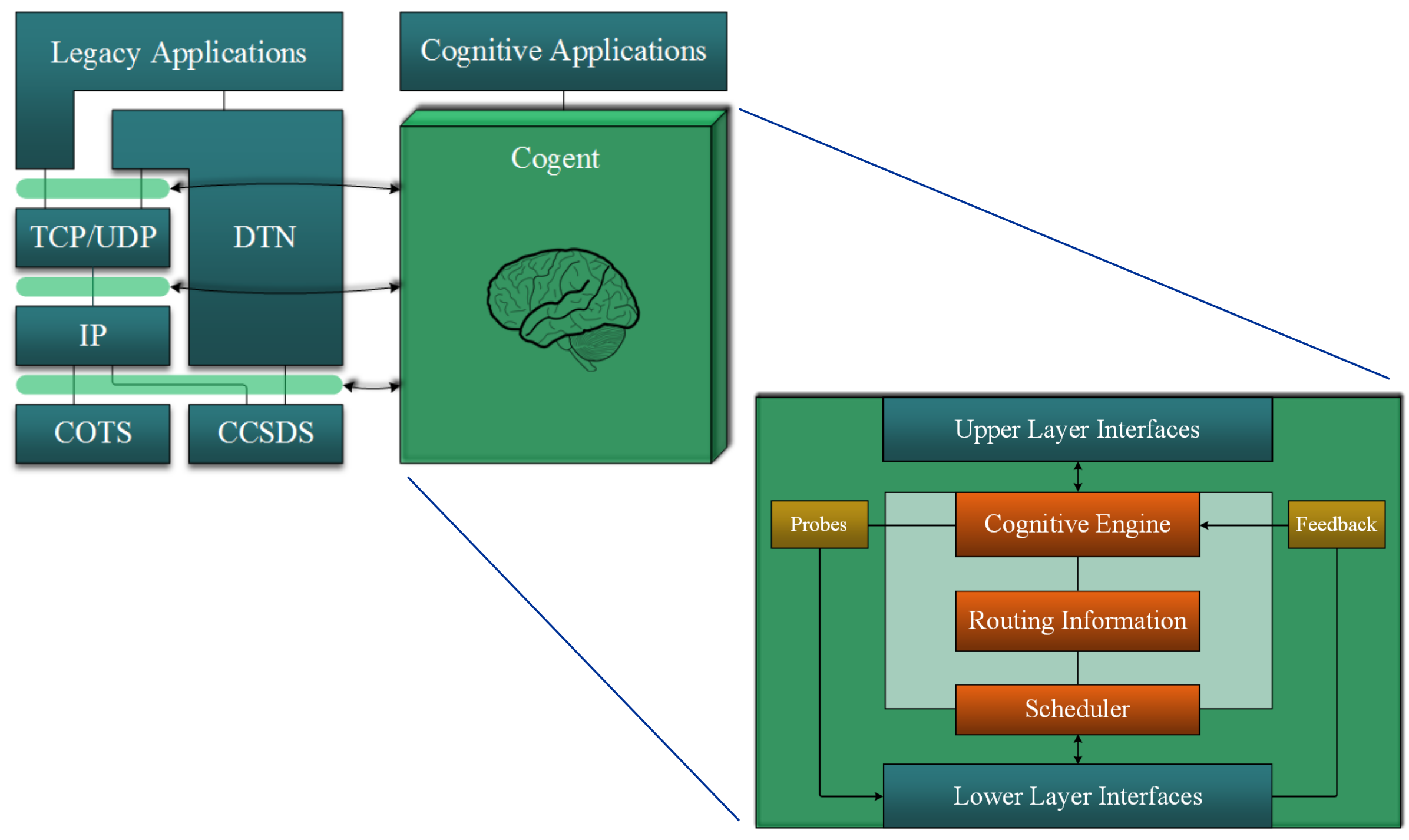




\section{Technology Gaps and Future Work}

- Cross-layer signaling

- Standardization is an important aspect of this ...

- Algorithm development

- More intelligent approaches to autonomy and management

- Computational offload

- Incremental upgrade of static hardware resources

- Debugging / management of intelligent systems

- "You did WHAT?! What were you THINKING?!"

- Self-knowledge

- "Generally, I'm not very good at ..."

- Self-design

- "Wouldn't it be nice if I could fly?" 


\section{Wrapping Things Up}

- Thanks for listening!

- Speaker: Gilbert Clark - gilbert.j.clark@nasa.gov

- Feel free to contact with questions, concerns, etc.

- Questions? Comments? Concerns? Criticisms? 